\title{
Idebenone reduces respiratory complications in patients with Duchenne muscular dystrophy
}

\author{
Craig M. McDonald ${ }^{\mathrm{a}}$, Thomas Meier ${ }^{\mathrm{b}}$, Thomas Voit ${ }^{\mathrm{c}, 1}$, Ulrike Schara ${ }^{\mathrm{d}}$, Chiara S.M. Straathof ${ }^{\mathrm{e}}$, \\ M. Grazia D’Angelo ${ }^{\mathrm{f}}$, Günther Bernert ${ }^{\mathrm{g}}$, Jean-Marie Cuisset ${ }^{\mathrm{h}}$, Richard S. Finkel ${ }^{\mathrm{i}}$, \\ Nathalie Goemans ${ }^{j}$, Christian Rummey ${ }^{k}$, Mika Leinonen ${ }^{k}$, Paolo Spagnolo ${ }^{b}$, \\ Gunnar M. Buyse ${ }^{\mathrm{j}, *}$ on behalf of the DELOS Study Group \\ ${ }^{a}$ University of California Davis Medical Center, Sacramento, CA, USA \\ b Santhera Pharmaceuticals, Liestal, Switzerland \\ 'Institut de Myologie, UPMC INSERM UMR 974, CNRS FRE 3617, Groupe Hospitalier de la Pitié Salpêtrière, Paris, France \\ ${ }^{\mathrm{d}}$ Universitätsklinikum, Essen, Germany \\ ${ }^{\mathrm{e}}$ LUMC, Leiden, The Netherlands \\ ${ }^{\mathrm{f}}$ IRCCS Eugenio Medea, Lecco, Italy \\ ${ }^{\mathrm{g}}$ G.v. Preyer'sches Kinderspital, Wien, Austria \\ ${ }^{\mathrm{h}} \mathrm{CHRU}$ de Lille, Lille, France \\ i Nemours Children's Hospital, Orlando, FL, USA \\ ${ }^{\mathrm{j}}$ University Hospitals Leuven, Leuven, Belgium \\ k 4Pharma, Liestal, Switzerland
}

Received 5 April 2016; received in revised form 10 May 2016; accepted 10 May 2016

\begin{abstract}
In Duchenne muscular dystrophy (DMD), progressive loss of respiratory function leads to restrictive pulmonary disease and places patients at significant risk for severe respiratory complications. Of particular concern are ineffective cough, secretion retention and recurrent respiratory tract infections. In a Phase 3 randomized controlled study (DMD Long-term Idebenone Study, DELOS) in DMD patients 10-18 years of age and not taking concomitant glucocorticoid steroids, idebenone $(900 \mathrm{mg} /$ day) reduced significantly the loss of respiratory function over a 1 -year study period. In a post-hoc analysis of DELOS we found that more patients in the placebo group compared to the idebenone group experienced bronchopulmonary adverse events (BAEs): placebo: 17 of 33 patients, 28 events; idebenone: 6 of 31 patients, 7 events. The hazard ratios (HR) calculated "by patient" (HR 0.33, p = 0.0187) and for "all BAEs" (HR 0.28, p=0.0026) indicated a clear idebenone treatment effect. The overall duration of BAEs was 222 days (placebo) vs. 82 days (idebenone). In addition, there was also a difference in the use of systemic antibiotics utilized for the treatment of BAEs. In the placebo group, 13 patients (39.4\%) reported 17 episodes of antibiotic use compared to 7 patients (22.6\%) reporting 8 episodes of antibiotic use in the idebenone group. Furthermore, patients in the placebo group used systemic antibiotics for longer (105 days) compared to patients in the idebenone group (65 days). This post-hoc analysis of DELOS indicates that the protective effect of idebenone on respiratory function is associated with a reduced risk of bronchopulmonary complications and a reduced need for systemic antibiotics.

(C) 2016 The Authors. Published by Elsevier B.V. This is an open access article under the CC BY-NC-ND license (http://creativecommons.org/ licenses/by-nc-nd/4.0/).
\end{abstract}

Keywords: Duchenne muscular dystrophy; Idebenone; Respiratory function; Airway infection

\section{Introduction}

Duchenne muscular dystrophy (DMD) is characterized by relentlessly progressive weakness and loss of muscle function,

\footnotetext{
* Corresponding author. Child Neurology, University Hospitals Leuven, Herestraat 49, B-3000 Leuven, Belgium. Tel.: +32 163438 45; fax: +32 16343842.

E-mail address: gunnar.buyse@uzleuven.be (G.M. Buyse).

${ }^{1}$ Present address: UCL Institute of Child Health; Great Ormond Street Hospital, London, UK.
}

cardiomyopathy, progressive spinal abnormality, restrictive lung disease and death in early adulthood [1]. Patients with DMD are at high risk of respiratory complications as their clinical condition deteriorates due to progressive loss of respiratory muscle strength [2,3]. Indeed, respiratory complications are a major cause of morbidity and mortality in DMD. It was previously estimated that $55-90 \%$ of DMD patients die from respiratory failure between 16.2 and 19 years of age $[4,5]$. 
For patients with DMD, neuromuscular weakness leads to impaired cough and airway clearance of secretions and acute respiratory failure may often occur in the setting of benign upper respiratory tract infections [6,7]. During these episodes, already severe pulmonary dysfunction is further compromised by bronchial mucus plugging and by further weakening and fatigue of inspiratory and expiratory muscles [4]. Such episodes can result in repeated pneumonias, hospitalizations, tracheal intubations, respiratory failure requiring ventilatory support and ultimately death. McDonald et al. reported that in a group of 114 DMD subjects, $34 \%$ had a history of significant respiratory complications such as pneumonia or acute respiratory failure and that there was an increase in the rate of complications with age [8]. This study further reported that there was a high correlation between pulmonary complications and forced vital capacity percent of predicted (FVC\%p) since 69\% of cases with $\mathrm{FVC} \% \mathrm{p}<50 \%$ had pulmonary complications compared with only $17 \%$ of those with $\mathrm{FVC} \% \mathrm{p}>80 \%$. While annual pulmonary evaluations are recommended for younger patients in the late first decade, as soon as the patient becomes non-ambulatory, more aggressive pulmonary monitoring and preventive interventions are recommended. According to internationally recognized guidelines, a structured approach to respiratory management of DMD comprises: (i) monitoring of respiratory function to detect respiratory involvement; (ii) introduction of lung volume recruitment techniques; (iii) use of a mechanical insufflation-exsufflation device to improve airway clearance and to reduce the risk of pulmonary infections; and (iv) the use of non-invasive ventilation (NIV) to prevent nocturnal hypoventilation, sleep disordered breathing, and ultimately respiratory failure [7,9-11]. Indeed, the use of non-invasive mechanical ventilation has increased survival in DMD from the late teens to the late 20 s $[12,13]$.

Respiratory muscle weakness of diaphragm and chest wall muscles, chest deformity resulting from progressive scoliosis, and fibrosis of chest wall muscles all contribute to a progressive restrictive pulmonary impairment in DMD, and predispose to a variety of pulmonary complications, which account for the majority of DMD-related deaths [14-17]. Of particular clinical concern is the high risk of recurrent respiratory tract infections and acute respiratory failure secondary to retained airway secretions, which, in turn, is due to reduced cough effectiveness caused by inspiratory, glottis and expiratory muscle weakness $[18,19]$. Specifically, in DMD peak cough flow (PCF) between 160 and $270 \mathrm{~L} / \mathrm{min}$ is considered sufficient for effective cough as long as the patient is in a stable medical condition. However, when the PCF falls below $160 \mathrm{~L} / \mathrm{min}$, the cough is no longer effective enough to provide adequate mucociliary clearance [4,20-22]. Similarly, reduced lung volumes measured as FVC below $1 \mathrm{~L}$ or inspiratory vital capacity (IVC) below $1.1 \mathrm{~L}$ has been associated with increased risk of severe chest infections, respiratory failure, and early morbidity in patients with DMD $[22,23]$. In addition, infections, including mild upper-respiratory tract infection, may worsen respiratory muscle weakness leading to a vicious cycle of recurrent, ever-worsening episodes of chest infection and inflammation, ineffective cough and excess mucus secretion and retention, which may result in atelectasis, ventilation-perfusion mismatch, hypoxemia and respiratory failure in severe cases [24]. The importance of maintaining effective cough and reducing the risk of airway infections has recently been quantitatively assessed by a patient and caregiver survey. In a community engaged approach conducted by Parent Project Muscular Dystrophy [25], 133 individuals, the majority of which represent DMD patients, rated effective cough and reduced airway infections as important treatment objectives for their disease [26].

In a Phase 3 randomized, placebo-controlled clinical study (DMD Long-term Idebenone Study, DELOS) in patients with DMD not using concomitant glucocorticoid steroids (GC), idebenone, a short-chain benzoquinone, has been shown to reduce the loss of respiratory function as assessed by peak expiratory flow (PEF), FVC and forced expiratory volume in 1 second (FEV1) over the 52-week study period [27]. In addition, idebenone was shown to reduce the loss of inspiratory lung function in the same trial [28]. Initial analyses of the DELOS study outcome already indicated an effect of idebenone treatment on the rate of airway infections. The aim of the present study was to further assess whether idebenone treatment as compared to placebo reduces the risk of bronchopulmonary adverse events (BAEs), assessed as proportion of patients experiencing such complications as well as their duration and associated hazard ratios. Furthermore, the use of antibiotics utilized for the treatment of such BAEs/airway infections was determined. The results from these analyses demonstrated that, in the setting of a placebo-controlled trial, idebenone use reduced the risk of experiencing BAEs, including airway infections, and reduced the need of systemic antibiotic use in patients with DMD.

\section{Methods}

\subsection{Patients}

The study population consisted of 64 DMD patients not using concomitant GCs enrolled in DELOS (ClinicalTrials.gov NCT01027884), a multi-center, Phase 3 randomized, doubleblind clinical trial comparing the efficacy and safety of $900 \mathrm{mg}$ /day idebenone (Raxone $^{\circledR}$ provided by Santhera Pharmaceuticals, Switzerland) vs. placebo [27]. DELOS was approved by competent national authorities responsible for the permission of clinical studies and IRBs/independent ethics committees and conducted according to GCP and the principles of the Declaration of Helsinki. Patients were enrolled in the study if they had a peak expiratory flow percent predicted $(\mathrm{PEF} \% \mathrm{p}) \leq 80 \%$ at baseline. Treatment duration was 52 weeks. In the intention-to-treat (ITT) population there were 31 patients randomized to idebenone and 33 patients randomized to placebo. The age of patients was $10-18$ years (mean age, 14.3 years) with patients in the placebo group on average being slightly older (15.0 years, SD 2.5) than patients in the idebenone group (13.5 years, SD 2.7). The vast majority $(92.2 \%)$ of patients were non-ambulatory at baseline and this was well balanced between treatment groups (idebenone: $90 \%$, placebo: 94\%). Overall, almost half of the patients (43.8\%) had never used GCs prior to study entry (idebenone: $45 \%$, placebo: 
$42 \%$ ) and all patients who previously used GCs had to have stopped their GC intake at least 12 months prior to study entry to be eligible. Patients were not allowed to take GCs during the 52 -week study period. Further details on study procedures and eligibility criteria have been reported previously [27].

\subsection{Respiratory function tests}

Standard spirometry was performed at hospital visits (i.e., baseline, Weeks 13, 26, 39 and 52) using a Pneumotrac Spirometer 6800 (Vitalograph, United Kingdom) with the aid of a qualified, trained and certified operator and in accordance with the American Thoracic Society/European Respiratory Society guidelines [29]. The assessment of respiratory function tests (including PEF and FVC) and conversion to percent predicted $(\% p)$ values to account for maturational changes is described in the original report of the DELOS study [27]. Specifically, body height was calculated from ulna length measurements.

\subsection{Assessment of bronchopulmonary adverse events and antibiotic use}

In a post-hoc efficacy analysis, treatment emergent adverse events (TEAEs) prospectively collected and recorded in the DELOS safety database were reviewed and assessed by a respiratory physician (Dr. Marianne Mann, Board certified pulmonologist, past Deputy Director for the Division of Pulmonary and Allergy Drug Products at the FDA) who was blinded to treatment assignment and had not been involved as an investigator in the DELOS trial.

Bronchopulmonary adverse events (BAEs) were defined as clinically relevant TEAEs if they involved the larynx, trachea, bronchi, lower airways or lung. Conversely, if the TEAEs were less precise and/or involved primarily the nose, sinuses, or throat, they were not considered BAEs. The following TEAEs (preferred terms) were identified as clinically relevant BAEs and included in the analysis: bronchitis, influenza (with respiratory symptoms), laryngitis, pneumonia, upper respiratory tract infection, viral infection (with respiratory symptoms), respiratory failure, acute respiratory failure, cough, and dyspnea. Conversely, TEAEs such as influenza-like illness, nasal congestion, nasopharyngitis, oropharyngeal pain, otitis media, pharyngitis, pyrexia, rhinitis, allergic rhinitis, rhinorrhea and sleep apnea syndrome were excluded from the analysis. BAEs were only assessed if they had been reported during the time of study medication intake. One event occurring before treatment start (dyspnea in the idebenone group) and two events occurring after the last dose of study medication intake ( 2 cases of bronchitis in 2 placebo patients) were excluded from the analysis.

The duration of the events was calculated from the starting date to the end date recorded in the safety database of the DELOS trial. If an event extended the period of study medication intake, the duration was only calculated until the last day of study medication intake. For events with unknown end date, the median length of events with identical preferred term was imputed. In cases where only the month was available, the 15th day of the month was used. Yearly incidence rates of BAEs by baseline pulmonary function were calculated as the number of events divided by exposure to study medicine in patient years.

The same study-independent assessor also determined the use of antibiotics listed as concomitant medications. For this, antibiotic use was classified by systemic exposure (e.g., excluding topical applications) and whether they were likely to be used for treatment of airway infections or related diseases, taking into consideration the indication(s) for which antibiotics were prescribed as reported by the investigator in the concomitant medication records. Systemic antibiotics in the vast majority of cases were amoxicillin/clavulanic acid or amoxicillin (generally oral).

\subsection{Statistical methods}

The hazard ratios for BAEs were calculated using a Cox Proportional Hazards model for idebenone over placebo using the day of the first event of a subject or the last day of study medication intake as censoring time if a patient had no events. Similarly, for all events a Proportional Means Regression model for recurrence data was used allowing the count of multiple events per patient [30,31]. All statistical analyses were of exploratory nature and nominal p-values are reported without adjustments for multiple testing.

\subsection{Role of the funding source}

The study was sponsored by Santhera Pharmaceuticals. The investigators and authors had sole discretion over study design, collection, analysis, and interpretation of data, writing of the report, and decision to submit it for publication.

\section{Results}

\subsection{Effect of idebenone on proportion of patients falling} below clinically relevant thresholds of respiratory function

In a pre-specified analysis of the DELOS trial, the proportion of patients falling below the clinically relevant threshold of $160 \mathrm{~L} / \mathrm{min}$ in PCF was assessed. In the ITT population there were 26 patients in the idebenone group and 33 patients in the placebo group who at baseline had a PCF of more than $160 \mathrm{~L} / \mathrm{min}$. During the 52 -week study period, only 1 patient $(3.8 \%)$ in the idebenone group compared to 6 patients $(18.2 \%)$ in the placebo fell below the threshold of $160 \mathrm{~L} / \mathrm{min}$ in PCF. We also analyzed the proportion of patients falling below $1 \mathrm{~L}$ in FVC. There were 9 patients in the placebo group and 6 patients in the idebenone group who at baseline had an FVC of more than $1 \mathrm{~L}$ but less than $1.5 \mathrm{~L}$ and who were therefore at risk of falling below the $1 \mathrm{~L} \mathrm{FVC} \mathrm{threshold.} \mathrm{Again} \mathrm{a} \mathrm{higher} \mathrm{number}$ of patients in the placebo group (5 patients, $55.6 \%$ ) fell below this threshold compared to patients in the idebenone group (1 patient, 16.7\%). When accounted for the entire ITT population, the proportion of patients falling below $1 \mathrm{~L}$ in $\mathrm{FVC}$ was $15.6 \%$ for the placebo group and $3.2 \%$ for the idebenone group. As these thresholds in PCF and FVC are clinically relevant risk factors for upper and lower airway infections and respiratory complications in DMD, we next assessed the DELOS study population for incidence of bronchopulmonary complications reported in the safety records of the study. 
Table 1

Analysis of bronchopulmonary adverse events (ITT population).

\begin{tabular}{|c|c|c|c|}
\hline & $\begin{array}{l}\text { Idebenone }(\mathrm{n}=31) \\
\text { Event count } \\
\text { [patient count] }\end{array}$ & $\begin{array}{l}\text { Placebo }(\mathrm{n}=33) \\
\text { Event count } \\
\text { [patient count] }\end{array}$ & $\begin{array}{l}\text { Fisher's } \\
\text { exact test* }\end{array}$ \\
\hline Bronchopulmonary adverse events & $7[6]$ & $28[17]$ & $\mathrm{p}=0.0096$ \\
\hline Upper respiratory tract infection & $2[2]$ & $10[6]$ & \\
\hline Bronchitis & $5[4]$ & $5[5]$ & \\
\hline Pneumonia & & $3[2]$ & \\
\hline Cough & & $2[2]$ & \\
\hline Influenza with respiratory symptoms & & $2[2]$ & \\
\hline Viral infection with respiratory symptoms & & $2[2]$ & \\
\hline Acute respiratory failure & & 1 & \\
\hline Dyspnea & & 1 & \\
\hline Laryngitis & & 1 & \\
\hline Respiratory failure & & 1 & \\
\hline
\end{tabular}

* Calculated for number of patients.

\subsection{Analysis of bronchopulmonary adverse events (BAE)}

The number and type of treatment-emergent BAEs in DELOS are summarized in Table 1. A total of 6 patients $(19.4 \%)$ in the idebenone group reported 7 BAEs compared to 17 patients $(51.5 \%)$ reporting 28 BAEs in the placebo group $(\mathrm{p}=0.0096$ for difference in patients experiencing BAEs). Time to event as well as frequency and duration of BAEs were further analyzed using a Cox Proportional Hazards model (first event only) and also Proportional Means Regression analysis (all events). The resulting cumulative frequency of all BAEs recorded during the one-year study period shows the lower rate of BAEs in the idebenone group compared to placebo (Fig. 1). The resulting hazard ratio from the Cox Proportional Hazards model for the number of patients experiencing at least one BAE was 0.327 (95\% CI: 0.129, 0.830; $p=0.0187$ ), which is in favor of idebenone treatment. Likewise, investigating all BAEs allowing for multiple events per patient by the Proportional Means Regression Model resulted in a hazard ratio of 0.281 (95\% CI: $0.123,0.642$; $\mathrm{p}=0.0026$ ), which is also in favor of idebenone treatment. In addition, the duration of treatment-emergent BAEs was analyzed by treatment group (Table 2). Although the mean duration of BAEs was slightly longer in the idebenone group compared to placebo, the median was comparable. The total number of days for which BAEs were reported in the idebenone group was 82 days, representing only $36.9 \%$ of the 222 days reported for the placebo group.

Interestingly, the higher frequency of BAEs in the placebo group was also reflected in the number of reported Serious Adverse Events (SAEs) leading to hospital admissions with respiratory causes. Such hospitalizations were reported for 4 patients in the placebo group and 1 patient in the idebenone group. Reasons for hospitalizations given for the placebo-treated patients were (i) acute respiratory failure, (ii) pneumonia, (iii) pulmonary micro-embolism following femur fracture and (iv) global respiratory failure. The patient from the idebenone group was hospitalized for sleep apnea syndrome.

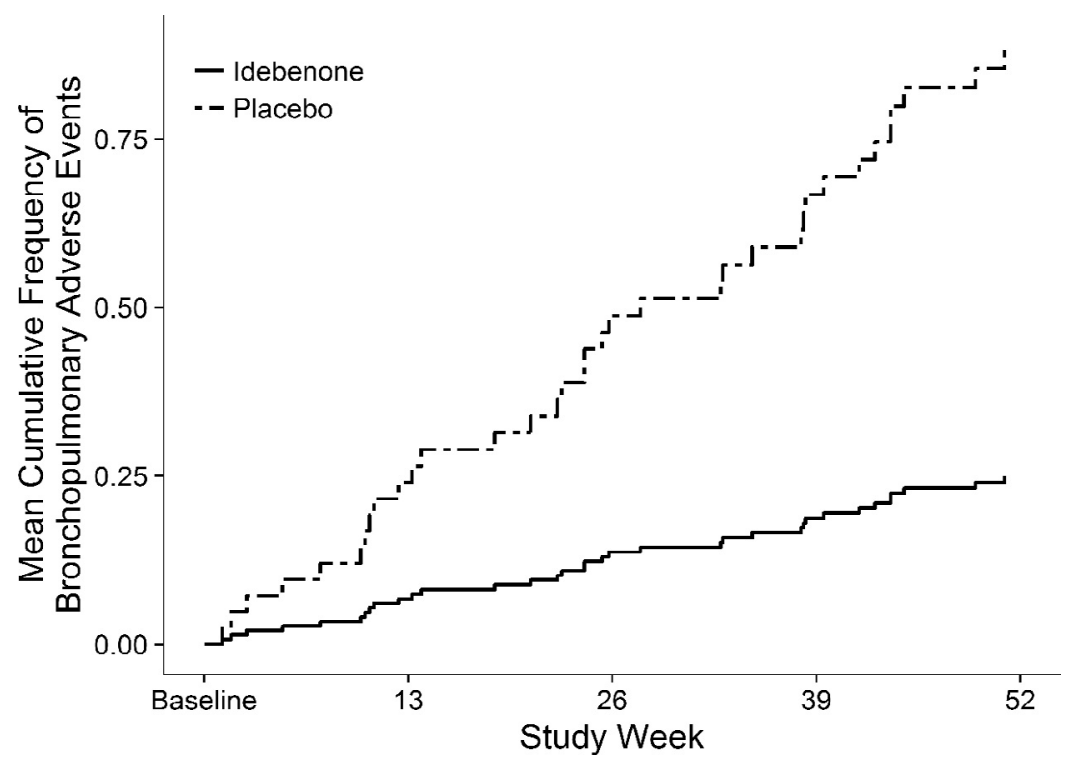

Fig. 1. Cumulative frequency for bronchopulmonary adverse events from baseline to Week 52 . 
Table 2

Duration of bronchopulmonary adverse events.

\begin{tabular}{|c|c|c|c|c|}
\hline $\begin{array}{l}\text { Treatment } \\
\text { group }\end{array}$ & $\begin{array}{l}\text { Total number } \\
\text { of BAEs }\end{array}$ & $\begin{array}{l}\text { Number } \\
\text { of patients }\end{array}$ & $\begin{array}{l}\text { Duration [days] } \\
\text { of BAEs* } \\
\text { Mean (SD); } \\
\text { median; range }\end{array}$ & $\begin{array}{l}\text { Total duration } \\
\text { [days] per } \\
\text { group }\end{array}$ \\
\hline Idebenone & 7 & 6 & $11.7(8.3) ; 9 ; 5-28$ & 82 \\
\hline Placebo & 28 & 17 & 7.9 (5.4); $8 ; 1-26$ & 222 \\
\hline
\end{tabular}

* Count of days for the period the patient has study medication.

Taken together, the data analyses performed provide consistent evidence favoring idebenone with regard to both frequency and duration of BAEs.

\subsection{Effect of age, baseline respiratory function and previous steroid use on BAEs}

We assessed the possible influence of age, previous glucocorticoid steroid use and respiratory function (measured as PEF\%p and FVC\%p) at baseline on the occurrence of BAEs. There was no marked difference in the age of patients with or without BAEs when compared within each treatment group (Table 3). However, the slightly older age in the placebo group in both patients with and without BAEs is reflecting the slight overall age imbalance between treatment groups. In each treatment group the age at baseline for patients with or without BAEs was comparable (idebenone: 12.3 years for patients with BAEs and 13.8 years for patients not reporting BAEs; placebo: 15.1 years for patients with BAEs and 14.9 years for patients not reporting BAEs).

Baseline respiratory function measured as PEF\%p was comparable and largely overlapping between all groups indicating that the difference in the number of BAEs between the idebenone and placebo groups was not driven by an imbalance in PEF\%p. On the other hand, there was a noticeable imbalance for $\mathrm{FVC} \% \mathrm{p}$ at baseline. For patients not experiencing BAEs the baseline $\mathrm{FVC} \% \mathrm{p}$ was comparable between placebo and idebenone groups. However, patients in the placebo group who experienced BAEs had lower baseline $\mathrm{FVC} \%$ p compared to patients in the idebenone group with BAEs.

To elucidate the impact of Baseline age, PEF\%p and $\mathrm{FVC} \% \mathrm{p}$, and prior $\mathrm{GC}$ use on the results, all factors were included in a multivariate Cox model. None of fixed factors showed statistically significant influence on the model (Age: $\mathrm{p}=0.5873 ;$ PEF\% $\mathrm{p}: \mathrm{p}=0.9336 ; \mathrm{FVC} \% \mathrm{p}: \mathrm{p}=0.9312$ ). Also

Table 3

Influence of age, baseline PEF\%p and baseline $\mathrm{FVC} \% \mathrm{p}$ on occurrence of BAEs.

\begin{tabular}{llrlll}
\hline $\begin{array}{l}\text { BAEs } \\
\text { reported }\end{array}$ & Treatment & $\mathrm{n}$ & $\begin{array}{l}\text { Baseline } \\
\text { age [year] }\end{array}$ & $\begin{array}{l}\text { Baseline } \\
\text { PEF\%p }\end{array}$ & $\begin{array}{l}\text { Baseline } \\
\text { FVC\%p }\end{array}$ \\
\hline Yes & Idebenone & 6 & $12.3(1.5)$ & $57.4(8.3)$ & $69.8(12.4)$ \\
Yes & Placebo & 17 & $15.1(2.2)$ & $53.7(12.9)$ & $47.8(18.0)$ \\
No & Idebenone & 25 & $13.8(2.9)$ & $52.5(10.6)$ & $51.9(14.7)$ \\
No & Placebo & 16 & $14.9(2.8)$ & $54.7(13.8)$ & $53.2(22.1)$ \\
\hline
\end{tabular}

Data are mean (SD). prior GC use did not show a significant influence on the results $(\mathrm{p}=0.6787)$. Analogously in a Proportional Means Regression model including the identical covariates, no influence of the fixed factors was shown (Age: $\mathrm{p}=0.2855$; PEF\%p: $\mathrm{p}=0.4677$; FVC\%p: $p=0.6913$; prior GC use: $p=0.3377$ ).

To further assess whether baseline pulmonary function was predictive for the occurrence of BAEs, we calculated the yearly rate of BAEs categorized by baseline $\mathrm{PEF} \% \mathrm{p}$ and $\mathrm{FVC} \% \mathrm{p}$. Fig. 2 shows the yearly rates of reported BAEs for patients separated into categories of $>80 \%,>60$ to $\leq 80 \%,>40$ to $\leq 60 \%$, and $\leq 40 \%$ of baseline $\mathrm{PEF} \% \mathrm{p}$ and $\mathrm{FVC} \% \mathrm{p}$, respectively. When analyzed for baseline $\mathrm{PEF} \% \mathrm{p}$ categories, yearly incidence rates of BAEs were always higher in the placebo group compared to the idebenone treatment group. Differences were particularly prominent for patients with PEF\%p of $\leq 60 \%$, with more than $1 \mathrm{BAE} /$ year reported for patients in the placebo group, while patients treated with idebenone experienced far fewer events $(0.26 \mathrm{BAEs} /$ year in the $>40$ to $\leq 60 \%$ PEF\%p category and none in patients with PEF\%p of $\leq 40 \%$ at baseline). Similarly, the yearly incidence rate of BAEs was consistently higher in the placebo group compared to idebenone for all patients with an $\mathrm{FVC} \% \mathrm{p}$ of $\leq 80 \%$ at baseline. Few patients in the study had $\mathrm{FVC} \%$ p $>80 \%$. Importantly, again, the largest difference in incidence of BAEs was seen in patients with more severe functional pulmonary impairment at baseline. Specifically, among patients who presented with a PEF\%p or FVC\%p at baseline of $\leq 40 \%$ only those in the placebo group experienced BAEs (on average more than $1 \mathrm{BAE} /$ year). In striking contrast, none of the patients in the idebenone group with $\leq 40 \%$ in $\mathrm{PEF} \% \mathrm{p}$ or $\mathrm{FVC} \% \mathrm{p}$ at baseline reported BAEs.

Finally, we also analyzed for a potential influence of previous glucocorticoid steroid use on the occurrence of BAEs. In the idebenone group 17 of 31 (55\%) patients reported previous GC use, which was similar to the proportion in the placebo group (19 of 33, 58\%) [27]. When we analyzed the influence of previous $\mathrm{GC}$ use as categorical factor in a Cox Model, previous GC use did not appear to influence significantly the time to first BAE ( $p=0.6787$, see above). This indicates that previous use of GCs stopped at least 12 months prior to study entry did not influence the susceptibility of patients to experience BAE.

\subsection{Effect of idebenone on antibiotic use}

As severe bronchopulmonary events frequently require intervention with antibiotic treatment [7], we also analyzed the number of patients using systemic antibiotics as well as the duration of antibiotic use. Overall, 7 patients (22.6\%) reported 8 episodes of antibiotic use for the treatment of bronchopulmonary adverse events/respiratory tract infections in the idebenone group compared to 13 patients (39.4\%) reporting 17 episodes of antibiotic use in the placebo group (Table 4). The mean and median duration of antibiotic use of approximately one week (6- 8 days) was comparable between treatment groups. The cumulative duration of antibiotic use was 105 days among patients on placebo, which was clearly longer compared to patients receiving idebenone (65 days). 


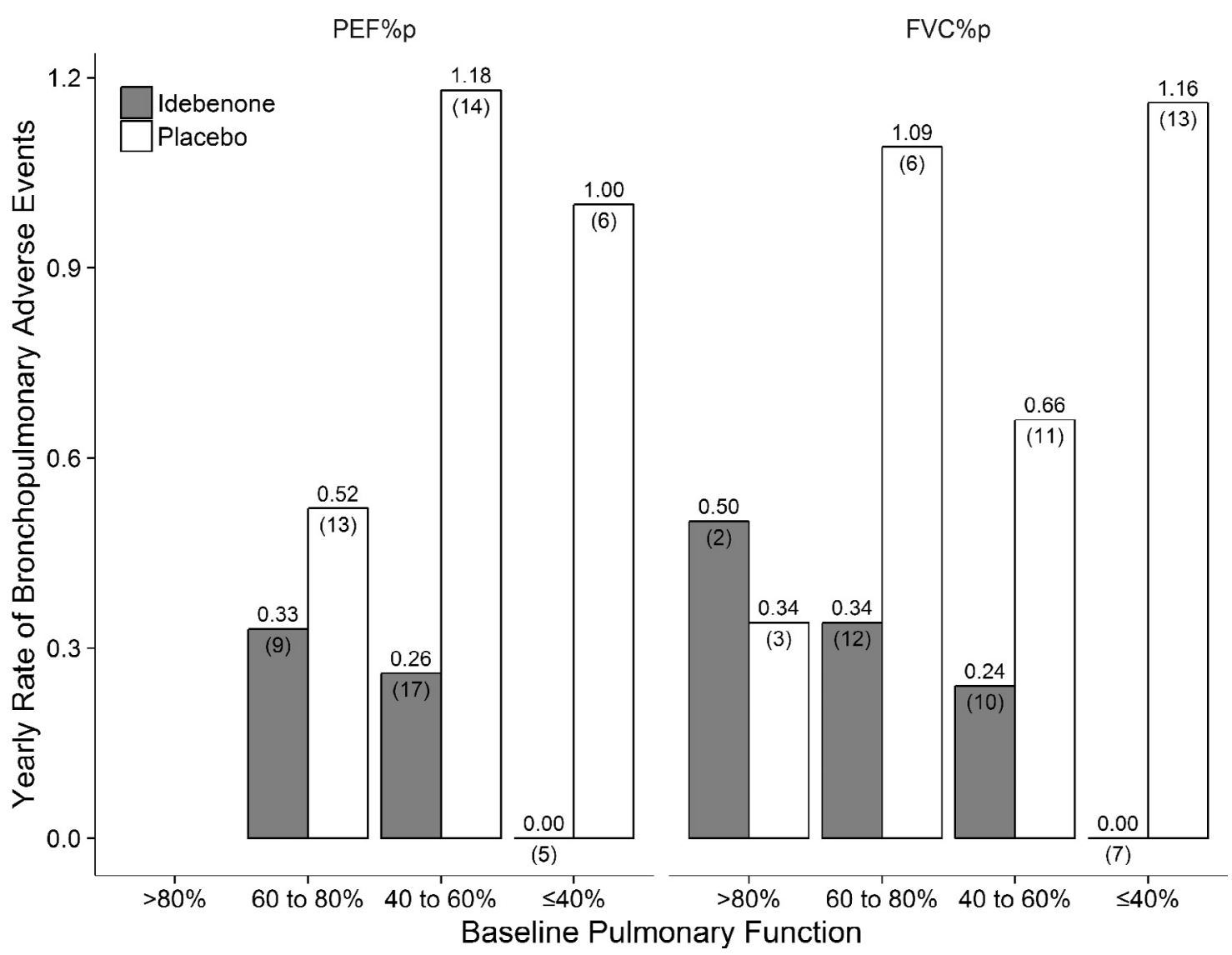

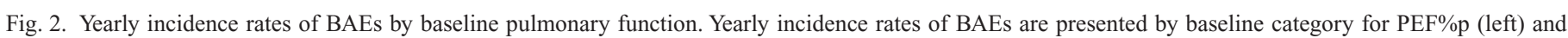

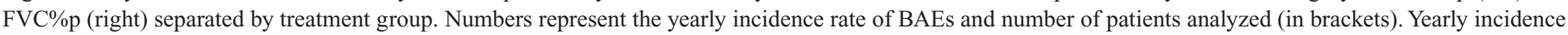

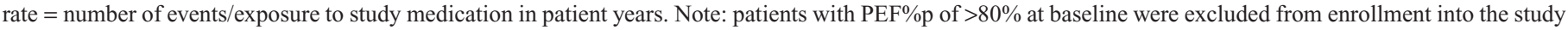
(exclusion criterion).

The resulting hazard ratio from the Cox Proportional Hazards model for time to first use of antibiotics was 0.52 (95\% CI: $0.207,1.304 ; p=0.1631$ ), favoring idebenone treatment. Likewise, investigating the cumulative frequency of the use of antibiotics (allowing for multiple use per patient) by the Proportional Means Regression Model resulted in a hazard ratio of $0.52(95 \% \mathrm{CI}: 0.226,1.217 ; \mathrm{p}=0.1330)$, which is also in favor of idebenone treatment (Fig. 3).

\section{Discussion}

In a Phase 3 randomized controlled clinical trial (DELOS) using pre-specified primary and secondary endpoints,

Table 4

Duration of antibiotic use for treatment of BAEs.

\begin{tabular}{|c|c|c|c|c|}
\hline $\begin{array}{l}\text { Treatment } \\
\text { group }\end{array}$ & Events* & Patients $^{\dagger}$ & $\begin{array}{l}\text { Duration [days] } \\
\text { of antibiotic use } \\
\text { Mean (SD); } \\
\text { median; range }\end{array}$ & $\begin{array}{l}\text { Total days }{ }^{\ddagger} \\
\text { of antibiotic } \\
\text { use }\end{array}$ \\
\hline Idebenone & 8 & 7 & $8.1(2.7) ; 8 ; 5-14$ & 65 \\
\hline Placebo & 17 & 13 & $6.2(2.1) ; 6 ; 2-11$ & 105 \\
\hline
\end{tabular}

* Events are defined as the number of periods of antibiotic use.

† Number of patients using antibiotics.

$\$$ Count of days for the period the patient has taken study medication. idebenone significantly reduced the loss of respiratory function in DMD patients $10-18$ years of age and not taking concomitant glucocorticoid steroids (GCs) over a 1-year study period. The current post-hoc analysis of the DELOS study showed that idebenone treatment compared to placebo reduced the rate of clinically relevant bronchopulmonary adverse events in patients with DMD. The beneficial effect of idebenone was similar irrespective of whether the data were analyzed by the number of patients experiencing these events or total number of events. Interestingly, the lower frequency of BAEs in the idebenone group was also reflected in a lower number of hospital admissions related to respiratory failure compared to the placebo group. A non-significant but clinically relevant trend favoring idebenone was also observed in terms of antibiotic use. Furthermore, idebenone reduced the proportion of patients falling below expert-defined clinically relevant thresholds of respiratory function associated with severely impaired airway clearance $(\mathrm{PCF}<160 \mathrm{~L} / \mathrm{min})$ [7] and risk of respiratory failure $(\mathrm{FVC}<1 \mathrm{~L})$ [23]. Only 1 patient $(3.8 \%)$ in the idebenone treated group compared to 6 patients $(18.2 \%)$ in the placebo fell below the threshold of $160 \mathrm{~L} / \mathrm{min}$ in PCF. The proportion of patients falling below $1 \mathrm{~L}$ in FVC over one-year was also reduced with idebenone treatment $(15.6 \%$ for the placebo group and $3.2 \%$ for the idebenone group). 


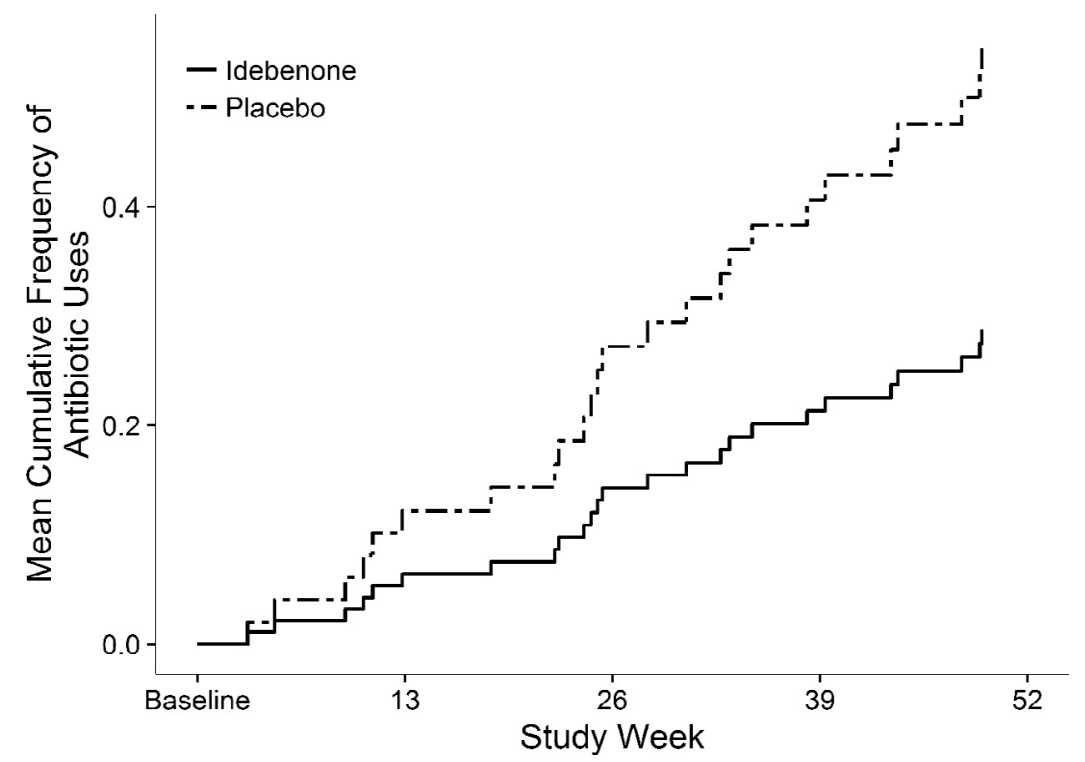

Fig. 3. Cumulative use of systemic antibiotics for treatment of BAEs from baseline to Week 52 .

Preservation of lung function and prevention and treatment of chest infections are among the most important aspects of the management of DMD. In this patient population, the increased risk of chest infections mainly results from weak cough and ineffective clearance of airway secretions. Several factors contribute to diminished effectiveness of cough in patients with DMD. Both the inspiratory and expiratory phases of cough are weakened, although the expiratory muscles are relatively more affected and provide for a large impact on the quality of cough [5]. Glottic dysfunction prevents the generation of adequate intrathoracic pressures needed to develop the sufficient shearing forces required for expectoration of airway secretions $[32,33]$. These factors together increase the risk of airway infections and pneumonia, which in patients with DMD can reach a rate of approximately one per year [19]. Impaired ability to clear secretions may also lead to life-threatening situations in which airways become obstructed by retained secretions. Mucus plugging not only reduces the lung parenchyma available for gas exchange, but also increases the risk of more severe infections, atelectasis and respiratory failure $[34,35]$. Notably, this vicious cycle can be triggered by relatively minor events, such as upper respiratory tract infections due to otherwise non-virulent viruses.

In DELOS idebenone reduced the yearly incidence rate of BAEs irrespective of baseline severity of pulmonary function impairment as assessed by PEF\%p and FVC\%p. However, its favorable effect was largest in patients with more advanced disease (i.e., lower PEF\%p and $\mathrm{FVC} \% \mathrm{p} \leq 60 \%$ ). Although the absolute value (or percent predicted) of the patient's FVC does not directly correlate with the quality of a patient's cough [5], the degree of restrictive lung disease provides a good representation of the patient's pulmonary reserve [36]. This can help determine whether (and which) respiratory support is needed [2,7]. In our analysis, neither age nor previous GC use influenced the occurrence of BAEs. Idebenone treatment was also associated with reduced antibiotic use in terms of both patients taking antibiotics and episodes of antibiotic use. While mean and median duration of antibiotic use was similar between groups, cumulative duration of antibiotic use was shorter in patients on idebenone. Finally, a trend favoring idebenone was also observed with regard to both the proportion of patients who used antibiotics at least once and cumulative antibiotic use allowing for multiple uses per patient.

While the use of non-invasive ventilation has improved survival in DMD by approximately 10 years, many individuals with DMD eventually die from complications of respiratory function decline, including progressive restrictive ventilatory defects, chronic hypoventilation and pulmonary infections. Therefore, prevention of these complications is a major goal of treatment. In agreement with previous analyses of the DELOS trial [27], the data presented here provide evidence that idebenone reduces the rate and duration of medical relevant BAEs and related antibiotic use compared to placebo in patients with DMD. These data provide evidence that the magnitude of the treatment benefits of idebenone seen with both expiratory and inspiratory respiratory function measures over one-year translates to clinically meaningful benefits. These tangible health benefits of more maintained effective cough and reduced airway infections have been noted by patients and caregivers to be important treatment objectives for their disease [26].

\section{Acknowledgments}

The study was sponsored by Santhera Pharmaceuticals. The DELOS Study Group is indebted to the participating patients and their parents. The authors thank Marianne Mann, MD (Regulatory Consultant, Highland, MD, USA) for categorizing BAEs and suggestions on the analyses. GMB is Senior Clinical Investigator of the Research Foundation-Flanders (FWO Vlaanderen, Belgium). 


\section{DELOS Study Group (sorted by country)}

Austria: G. Bernert, F. Knipp (Vienna). Belgium: G.M. Buyse, N. Goemans, M. van den Hauwe (Leuven). France: T. Voit, V. Doppler, T. Gidaro (Paris); J.-M. Cuisset, S. Coopman (Lille). Germany: U. Schara, S. Lutz (Essen); J. Kirschner, S. Borell, M. Will (Freiburg). Italy: M.G. D’Angelo, E. Brighina, S. Gandossini (Lecco); K. Gorni, E. Falcier (Milan); L. Politano, P. D'Ambrosio, A. Taglia (Naples); The Netherlands: J.J.G.M. Verschuuren, C.S.M. Straathof (Leiden). Spain: J.J. Vílchez Padilla, N. Muelas Gómez (Valencia). Sweden: T. Sejersen, M. Hovmöller (Stockholm). Switzerland: P.-Y. Jeannet, C. Bloetzer (Lausanne). USA: S. Iannaccone, D. Castro (Dallas); G. Tennekoon, R. Finkel, C. Bönnemann (Philadelphia); C. McDonald, E. Henricson, N. Joyce (Sacramento); S. Apkon, R.C. Richardson (Seattle).

\section{References}

[1] Bushby K, Finkel R, Birnkrant DJ, et al.; DMD Care Considerations Working Group. Diagnosis and management of Duchenne muscular dystrophy, part 1: diagnosis, pharmacological and psychosocial management. Lancet Neurol 2010;9:77-93.

[2] Bushby K, Finkel R, Birnkrant DJ, et al.; DMD Care Considerations Working Group. Diagnosis and management of Duchenne muscular dystrophy, part 2: implementation of multidisciplinary care. Lancet Neurol 2010;9:177-89.

[3] Khirani S, Ramirez A, Aubertin G, et al. Respiratory muscle decline in Duchenne muscular dystrophy. Pediatr Pulmonol 2014;49:473-81.

[4] Bach JR, Ishikawa Y, Kim H. Prevention of pulmonary morbidity for patients with Duchenne muscular dystrophy. Chest 1997;112:1024-8.

[5] Gozal D. Pulmonary manifestations of neuromuscular disease with special reference to Duchenne muscular dystrophy and spinal muscular atrophy. Pediatr Pulmonol 2000;29:141-50.

[6] Miske LJ, Hickey EM, Kolb SM, Weiner DJ, Panitch HB. Use of the mechanical in-exsufflator in pediatric patients with neuromuscular disease and impaired cough. Chest 2004;125:1406-12.

[7] Birnkrant DJ, Bushby KM, Amin RS, et al. The respiratory management of patients with Duchenne muscular dystrophy: a DMD care considerations working group specialty article. Pediatr Pulmonol 2010;45:739-48.

[8] McDonald CM, Abresch RT, Carter GT, et al. Profiles of neuromuscular disease. Duchenne muscular dystrophy. Am J Phys Med Rehabil 1995;74(Supp1.):S70-92.

[9] LoMauro A, D'Angelo MG, Aliverti A. Assessment and management of respiratory function in patients with Duchenne muscular dystrophy: current and emerging options. Ther Clin Risk Manag 2015;11:147588.

[10] Finder JD, Birnkrant D, Carl J, et al. Respiratory care of the patient with Duchenne muscular dystrophy: ATS consensus statement. Am J Respir Crit Care Med 2004;170:456-65.

[11] Finsterer J. Cardiopulmonary support in Duchenne muscular dystrophy. Lung 2006;184:205-15.

[12] Rall S, Grimm T. Survival in Duchenne muscular dystrophy. Acta Myol 2012;31:117-20.

[13] Passamano L, Taglia A, Palladino A, et al. Improvement of survival in Duchenne Muscular Dystrophy: retrospective analysis of 835 patients. Acta Myol 2012;31:121-5.

[14] Vianello A, Bevilacqua M, Salvador V, Cardaioli C, Vincenti E. Long-term nasal intermittent positive pressure ventilation in advanced Duchenne's muscular dystrophy. Chest 1994;105:445-8.
[15] Baydur A, Gilgoff I, Prentice W, Carlson M, Fischer DA. Decline in respiratory function and experience with long-term assisted ventilation in advanced Duchenne's muscular dystrophy. Chest 1990;97:884-9.

[16] Smith PE, Calverley PM, Edwards RH, Evans GA, Campbell EJ. Practical problems in the respiratory care of patients with muscular dystrophy. N Engl J Med 1987;316:1197-205.

[17] Inkley SR, Oldenburg FC, Vignos PJ Jr. Pulmonary function in Duchenne muscular dystrophy related to stage of disease. Am J Med 1974;56:297-306.

[18] Szeinberg A, Tabachnik E, Rashed N, et al. Cough capacity in patients with muscular dystrophy. Chest 1988;94:1232-5.

[19] Bach JR, Rajaraman R, Ballander F, et al. Neuromuscular ventilator insufficiency: effect of home mechanical ventilator use $\mathrm{v}$ oxygen therapy on pneumonia and hospitalization rates. Am J Phys Med Rehabil 1998;77:8-19.

[20] Gauld LM, Boynton A. Relationship between peak cough flow and spirometry in Duchenne muscular dystrophy. Pediatr Pulmonol 2005;39:457-60.

[21] Tzeng AC, Bach JR. Prevention of pulmonary morbidity for patients with neuromuscular disease. Chest 2000;118:1390-6.

[22] Dohna-Schwake C, Ragette R, Teschler H, Voit T, Mellies U. Predictors of severe chest infections in pediatric neuromuscular disorders. Neuromuscul Disord 2006;16:325-8.

[23] Phillips MF, Quinlivan RC, Edwards RH, Calverley PM. Changes in spirometry over time as a prognostic marker in patients with Duchenne muscular dystrophy. Am J Respir Crit Care Med 2001;164:2191-4.

[24] Simonds AK. Respiratory complications of the muscular dystrophies. Semin Respir Crit Care Med 2002;23:231-8.

[25] Peay HL, Hollin I, Fischer R, Bridges JF. A community-engaged approach to quantifying caregiver preferences for the benefits and risks of emerging therapies for Duchenne muscular dystrophy. Clin Ther 2014;36:62437.

[26] Parent Project Muscular Dystrophy [Internet]. PPMD submits results of patient-centered benefit-risk assessment study in Duchenne \& Becker to FDA. <http://community.parentprojectmd.org/profiles/blogs/ ppmd-submits-results-of-patient-centered-benefit-risk-assessment-?xg _source=Connect_news $>; 2015$ [accessed 11.03.16].

[27] Buyse GM, Voit T, Schara U, et al.; DELOS Study Group. Efficacy of idebenone on respiratory function in patients with Duchenne muscular dystrophy not using glucocorticoids (DELOS): a double-blind randomised placebo-controlled phase 3 trial. Lancet 2015;385:1748-57.

[28] Buyse GM, Voit T, Schara U, et al.; DELOS Study Group. Treatment effect of idebenone on inspiratory function in patients with Duchenne muscular dystrophy. Pediatr Pulmonol (submitted for publication)

[29] American Thoracic Society/European Respiratory Society. ATS/ERS statement on respiratory muscle testing. Am J Respir Crit Care Med 2002;166:518-624.

[30] Nelson WB. Recurrent events data analysis for products repairs, disease recurrences, and other applications (ASA-SIAM series on statistics and applied probability). Society for Industrial and Applied Mathematics; 2003.

[31] Lin DY, Wei LJ, Yang I, Ying Z. Semiparametric regression for the mean and rate functions of recurrent events. J R Stat Soc B 2000;62:711-30.

[32] Boitano LJ. Management of airway clearance in neuromuscular disease. Respir Care 2006;51:913-22.

[33] Panitch HB. Respiratory issues in the management of children with neuromuscular disease. Respir Care 2006;51:885-93.

[34] Schmidt-Nowara WW, Altman AR. Atelectasis and neuromuscular respiratory failure. Chest 1984;85:792-5.

[35] Hoffman LA. Ineffective airway clearance related to neuromuscular dysfunction. Nurs Clin North Am 1987;22:151-66.

[36] Kravitz RM. Airway clearance in Duchenne muscular dystrophy. Pediatrics 2009;123(Suppl. 4):S231-5. 